\title{
COMBINAÇÃO LINEAR PONDERADA NA DEFINIÇÃO DE ÁREAS PRIORITÁRIAS Â CONECTIVIDADE ENTRE FRAGMENTOS FLORESTAIS EM AMBIENTE SIG ${ }^{1}$
}

\author{
Anderson Antônio da Conceição Sartori², Ramon Felipe Bicudo da Silva ${ }^{3}$ e Célia Regina Lopes \\ Zimback $^{4}$
}

\begin{abstract}
RESUMO - A fragmentação florestal de origem antrópica é um dos resultados do processo desordenado de uso e ocupação do solo, especialmente em paisagens intensamente cultivadas. Neste contexto, o presente trabalho objetivou definir áreas prioritárias, para favorecer a conectividade entre os fragmentos florestais, visando ações de recuperação florestal na Bacia do Rio Pardo, SP, utilizando a abordagem multicriterial denominada Combinação Linear Ponderada.Na definição dos critérios e, posteriormente, dos pesos de fatores, empregou-se a Técnica Participatória. Os fatores considerados importantes ao objetivo do trabalho foram: proximidade entre fragmentos de maior área nuclear, proximidade da cobertura florestal, proximidade da rede hidrográfica, distância aos centros urbanos, declividade, erodibilidade do solo. Considerando que as variáveis que interferem na escolha de áreas prioritárias à restauração florestal na Bacia do Rio Pardo-SP contribuem com pesos diferenciados no processo final de decisão, estabeleceu-se uma hierarquia, de acordo com a importância de cada fator para a aptidão da área. O fator de maior peso foi proximidade entre fragmentos de maior área nuclear $(0,3713)$, seguido de proximidade da cobertura florestal $(0,1911)$, proximidade da rede hidrográfica $(0,1516)$, distância aos centros urbanos $(0,1168)$, declividade $(0,0840)$ e erodibilidade $(0,0854)$.O resultado obtido foi um mapa de áreas prioritárias, com cinco graus de prioridade. A priorização de áreas ocorreu de maneira a promover, primeiro a união dos fragmentos de floresta com maior área nuclear e, a partir dessa união, a sucessiva expansão dessas regiões de prioridade muito alta tendendo a muito baixa. A metodologia mostrou-se adequada ao mapeamento de áreas prioritárias à restauração florestal, em bacias hidrográficas. Uma vez que fragmentos com maiores áreas nucleares sejam conectados com fragmentos pequenos, onde estes são predominantes na paisagem, estes promoverão a formação de fragmentos maiores a partir da formação de corredores florestais e da recomposição da vegetação.
\end{abstract}

Palavras-chave: Bacia Hidrográfica, Avaliação Multicriterial, Restauração Florestal e Conectividade Florestal.

\section{WEIGHTED LINEAR COMBINATIONOF A GIS ENVIRONMENT IN THE DEFINITION OF PRIORITY AREAS FOR CONNECTIVITY AMONG FOREST FRAGMENTS}

\begin{abstract}
Forest fragmentation of anthropogenic origin is one of the results promoted by the disorderly process of the land use and land cover, especially in intensively cultivated landscapes. In this context, this study aimed to define priority areas to facilitate connectivity among forest fragments, seeking forest recovery actions in the Pardo River Basin, SP, using the multicriteria approach (Weighted Linear Combination). The Participator Technique was used to define the criteria and the weights of the factors. The factors considered important to the objective of this study were the following: proximity between the fragments with greater
\end{abstract}

\footnotetext{
${ }^{1}$ Recebido em 15.03.2012 aceito para publicação em 04.06.2012

${ }^{2}$ Pós-Graduação em Agronomia - Irrigação e Drenagem, Faculdade de Ciências Agronômicas-UNESP, Departamento de Ciência do Solo Recursos Naturais. E-mail: <sartori80@gmail.com>.

${ }^{3}$ Pós-Graduação em Ambiente e Sociedade, Universidade Estadual de Campinas, UNICAMP, Brasil. E-mail: $<$ ramonbicudo@yahoo.com.br>.

${ }^{4}$ Universidade Estadual Paulista Júlio de Mesquita Filho,UNESP. E-mail: <czimback@ gmail.com >.
} 
nuclear areas, proximity to forest cover, proximity of the river system, distance to urban centers, slope, erodibility of soil. Whereas the variables that influence the selection of priority areas for forest restoration in the Pardo River Basin, SP contribute with different weights in the final decision process, a hierarchy according to the importance of each factor to the fitness area was established. The major factor was proximity among fragments of the largest nuclear area (0.3713), followed by proximity to forest cover (0.1911), proximity to the river system (0.1516), distance to urban centers (.1168), slope (0.0840) and erodibility (0.0854). The result was a map of priority areas, with five classes of priority. The priority areas occurred in order to promote the first union of forest patches with greater nuclear area, and from this union, the successive expansion of these regions of very high priority tending to very low. The methodology was adequate to the mapping of priority areas for restoration in watersheds. Fragments with bigger nuclear areas, connected with small fragments where they are predominant in the landscape, promoting the formation of larger fragments from the formation offorest corridors and recompositon of vegetation.

Keywords: Watershed, Multicriteria Evaluation, Forest Restoration and Connectivity Forest

\section{1 - INTRODUÇÃO}

A Bacia do Rio Pardo-SP representa muito bem a situação de grande parte do território brasileiro originalmente coberto pela Mata Atlântica, onde a fragmentação florestal é um dos resultados do processo desordenado de uso e ocupação do solo, especialmente em paisagens intensamente cultivadas (PUTZ et al., 2011).

A conservação e restauração em fragmentos florestais representam grande desafio nos dias atuais em razão do elevado nível de perturbações antrópicas nos ecossistemas naturais. A maior parte dos remanescentes florestais, especialmente em paisagens intensamente cultivadas, encontra-se na forma de pequenos fragmentos, altamente perturbados, isolados, pouco conhecidos e pouco protegidos (VIANA, 1995). A fragmentação florestal de origem antrópica tem sido uma das principais causas de alteração, tanto na estrutura quanto nos processos de diferentes paisagens (LORD e NORTON, 1990; PUTZ et al., 2001).

A alternativa que se coloca é restaurar os fragmentos e interligá-los a corredores e sistemas agroflorestais de alto fluxo de biodiversidade. Ao recuperar os fragmentos (frequentemente degradados pela ação antrópica), aumenta-se o seu potencial como "ilhas de biodiversidade". Ao interligar os fragmentos através de "corredores de biodiversidade", aumentam-se o fluxo de animais e sementes e, portanto, a colonização das áreas degradadas pelas espécies de plantas e animais presentes nos fragmentos florestais. O desenvolvimento de tecnologias de recuperação de fragmentos degradados e o estabelecimento de corredores florestais e paisagens de maior porosidade constituem um dos maiores desafios para as atividades de restauração em paisagens fragmentadas (VIANA e PINHEIRO, 1998).

Na tentativa de garantir a manutenção da estrutura e dos processos ecológicos dos remanescentes florestais em paisagens, tem sido dada muita atenção às ações que buscam a determinação de áreas prioritárias para doção de práticas de restauração ecológica. A determinação dessas áreas prioritárias é o primeiro passo para a elaboração de estratégia regional ou nacional para a conservação da diversidade biológica, pois permite ordenar os esforços e recursos disponíveis para conservação e subsidiar a elaboração de políticas públicas de ordenamento territorial (SARTORI, 2010).

No que se refere à priorização de áreas, a espacialização representa um dos métodos mais eficientes e econômicos no manejo de bacias hidrográficas. O objetivo é alcançado quando há integração com os diferentes planos de informação da paisagem e suas características e, ou, processos, juntamente com os Sistemas de Informações Geográficas SIGs (VALENTE, 2005).

Malczewski (2006) comentou que, em muitas das aplicações de SIG na área de análise ambiental, é comum o envolvimento de múltiplos critérios para se atender a um ou mais objetivos. É a denominada Avaliação Multicritérios (AMC). Problemas de decisão espacial normalmente envolvem grande conjunto de alternativas viáveis e múltiplas em ambiente SIG, a AMC, por meio de diversas abordagens, como a Booleana, a Combinação Linear Ponderada (CLP) e a Média Ponderada Ordenada (MPO), que tem sido utilizada nas mais variadas aplicações (SARTORI, 2010).

Revista Árvore, Viçosa-MG, v.36, n.6, p.1079-1090, 2012 
Sartori e Zimback (2011) empregaram a Avaliação Multicritérios em ambiente SIG, para geração de um mapa de áreas prioritárias à recomposição florestal, visando à conservação de recursos hídricos. Wang e Medley (2004) também se valeram da tecnologia SIG para desenvolver um modelo baseado em múltiplos critérios, visando à identificação de áreas apropriadas à restauração florestal em Ohio, EUA, com relação ao seu potencial para conservação de carbono. Ferraz e Vettorazzi (2003) utilizaram AMC para identificação de áreas apropriadas à recomposição florestal, com espécies nativas, em fazendas de reflorestamentos, com base em princípios de ecologia da paisagem. Valente (2005) definiu áreas prioritárias para conservação e preservação florestal, tendo em vista o incremento da biodiversidade regional. Vettorazzi (2006) utilizou a Avaliação multicritérios em ambiente SIG para definir áreas prioritárias à restauração florestal, visando à conserva de recursos hídricos.

Nesse contexto, este trabalho objetivou definir áreas prioritárias para favorecer a conectividade entre os fragmentos florestais, com vistas a ações de recuperação florestal na Bacia do Rio Pardo-SP, utilizando a abordagem multicriterial (Combinação Linear Ponderada).

\section{MATERIAL E MÉTODOS}

\section{1. Área de Estudo}

A Bacia do Rio Pardo possui 148,76 km² e localiza-se na região Centro Sul do Estado de São Paulo, abrangendo áreas dos Municípios de Botucatu e Pardinho. O rio Pardo, seu principal curso de água, percorre um trecho de aproximadamente $28,7 \mathrm{~km}$, desde a sua nascente até o ponto de captação de água pela SABESP (CONTE, 1999). Esse rio é afluente direito do Paranapanema, que por sua vez integra a Bacia Hidrográfica do Rio Paraná. A Bacia do Rio Pardo está geograficamente localizada entre as coordenadas $23^{\circ} 06^{\prime} 14^{\prime \prime}$ e $22^{\circ} 56^{\prime} 07^{\prime \prime}$ de latitude sul e $48^{\circ} 28^{\prime} 37^{\prime \prime}$ e $48^{\circ} 20^{\prime} 40^{\prime \prime}$ de longitude oeste de Greenwich, com altitudes variando entre 840 e $1.000 \mathrm{~m}$.

Vários municípios da região, entre os quais Botucatu e Pardinho, bem como parte da área da Bacia do Rio Pardo, encontram-se na APA de Botucatu (Área de Proteção Ambiental), criada pelo Decreto Estadual n. ${ }^{\circ}$ 20.960, de 8 de junho de 1983. O objetivo da APA é proteger os recursos naturais de áreas compreendidas nas três regiões fisiográficas, sendo elas: Depressão Periférica, Frente e Reverso da "Cuesta" de Botucatu (SOUZA et al., 1985).

Com relação ao clima, a região pode ser enquadrada como tendo clima mesotérmico, identificado como Cwa subtropical úmido, segundo a classificação de Köppen, com inverno seco e verão chuvoso e quente, com temperaturas médias anuais em torno de $20^{\circ} \mathrm{C}$ e índice pluviométrico entre 1.100 e $1.700 \mathrm{~mm}$ anuais (CARVALHO e JIM, 1983).

A área em estudo encontra-se no Planalto Ocidental Paulista, com relevo uniforme, extensos espigões de perfis convexos e cimos ondulados, com terminações laterais lobadas, constituindo baixas e amplas colinas que avançam em direção aos vales dos principais rios. Nos locais onde os cursos d'água escavam seu vale, descobrindo a Formação Serra Geral, o relevo é mais acidentado, convexo, e os leitos dos rios são mais declivosos, o que torna as águas rápidas, sendo comum o aparecimento de saltos e cachoeiras (ALMEIDA, 1964).

De acordo com o levantamento pedológico semidetalhado da Bacia do Rio Pardo, escala 1:10.000, adaptado de Zimback (1997) por Grossi (2003), a bacia tem sua área ocupada com Latossolo Vermelho distrófico, Argissolo Vermelho-Amarelo distrófico, Neossolo Lítólico distrófico, Gleissolo Háplico e Latossolo Vermelho distroférrico.

Do ponto de vista da vegetação, a biodiversidade é expressiva. Percebeu-se a presença de matas de transição e atlântica, vegetação de Cerrado e campo cerrado, contando ainda, segundo Tornero (1996), com indivíduos isolados do pinheiro-do-paraná (Araucaria brasiliensis), testemunho de um clima pretérito mais frio.

\subsection{Uso e cobertura do solo}

O mapa de uso e cobertura do solo foi produzido pelo processamento digital das imagens orbitais. Foi feita a correção geométrica utilizando o modelo de transformação polinomial de primeiro grau e o método de interpolação pelo vizinho mais próximo. Nessa correção houve a identificação e registro das coordenadas de pontos, denominados controle, que foi comum entre as imagens digitais CBERS 2B, além de uma imagem do satélite LANDSAT-5 que, por sua vez, vez, representou as coordenadas reais.

Revista Árvore, Viçosa-MG, v.36, n.6, p.1079-1090, 2012 
As classes de uso e cobertura do solo foram definidas a partir do conhecimento de campo e pontos coletados com GPS.

\subsection{Método da Combinação Linear Ponderada}

Um dos métodos mais empregados na Avaliação Multicritérios (AMC) é a Combinação Linear Ponderada (CLP) (VOOGD, 1983). Uma vez que os mapas de critérios (fatores e restrições) tenham sido gerados, é uma simples questão de multiplicar cada mapa de fator (isto é, cada célula, ou pixel, de cada mapa) pelo seu peso e, então, somar os resultados. Devido ao fato de os pesos terem de somar 1, o mapa de adequação resultante terá variação de valores como aqueles dos mapas de fatores padronizados que foram usados, como mostrado na Figura 1.

\subsection{Mapas de fatores}

Para elaboração dos fatores foram utilizados planos de informações: mapa de solos, uso e cobertura do solo, fragmentos de floresta, declividade do terreno, rede hidrográfica e centros urbanos, provenientes de Sartori (2010).

Na definição dos critérios e, posteriormente, dos pesos de fatores, empregou-se a Técnica Participatória proposta por Eastman (2001), Malczewski (1999), Chen et al. (2000) e Valente (2005), que se constitui na reunião e consulta a especialistas das diferentes áreas de interesse do trabalho. Para aplicação dessa técnica, foi feita uma reunião no Grupo de Estudos e Pesquisas Agrárias

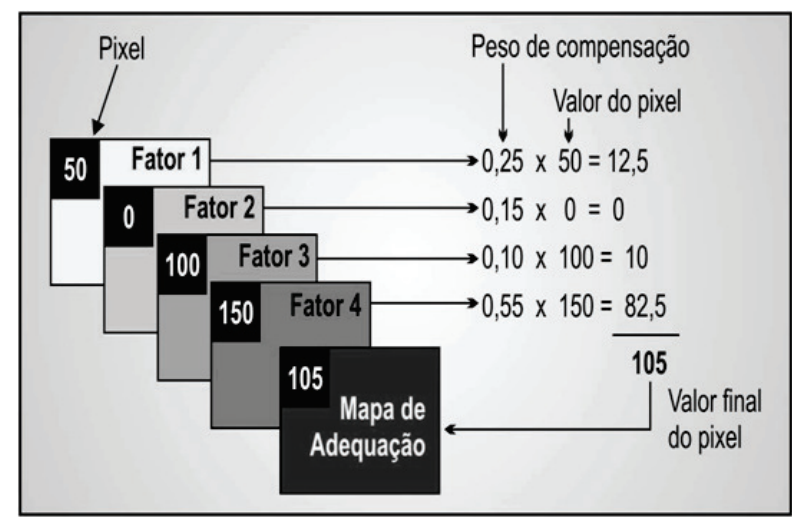

Figura 1 - Representação do método da Combinação Linear Ponderada (CLP).

Figure 1 -Representation of the method of Weighted Linear Combination (WLC).
Georreferenciadas (GEPAG) da Faculdade de Ciências Agronômica FCA/UNESP, que conta com vários integrantes de diversas formações, bem como consultaram-se outros pesquisadores de diferentes áreas do conhecimento. Dessa forma, todos os especialistas contribuíram com o projeto, sugerindo fatores (características/atributos da paisagem) e explicando o motivo dessas escolhas, que foram importantes para os objetivos do trabalho.

Ao final do processo de revisão de literatura, a avaliação de projetos desenvolvidos e da Técnica Participatória foram elencados seis fatores, juntamente com as áreas restritivas, de acordo com o objetivo de definir áreas prioritárias para restauração florestal, visando ao aumento da conectividade em fragmentos da Bacia do Rio Pardo. Os fatores considerados importantes foram elaborados de forma que cada mapa foi associado ao limite da área de estudo, para se terem essas distâncias somente dentro da área da bacia. Os fatores foram os seguintes, de acordo com o objetivo do trabalho:

(1) Proximidade entre fragmentos de maior área nuclear: a elaboração deste fator foi estabelecida de acordo com Valente (2005), por meio da seleção do Plano de Informação (PI) de fragmentos florestais, que quando descontados de determinada área considerada como borda ainda apresentam área nuclear. Para o cálculo das métricas, considerou-se uma borda de $50 \mathrm{~m}$ para cada fragmento de mata. Essa borda, segundo Murcia (1995), é a distância na qual os efeitos de borda tendem a desaparecer gradativamente em direção à área nuclear.

Fragmentos com maiores áreas nucleares e conectados compõem a classe de maior prioridade no mapa final de áreas prioritárias, para favorecer a conectividade entre os fragmentos florestais e são tipicamente melhores para a conservação da biodiversidade, de acordo com Noss et al. (1997). Eles são, ainda segundo Geneletti (2004), os componentes básico da estrutura de uma paisagem que visa manter a integridade de sua cobertura florestal natural.

Primeiramente, os fragmentos foram divididos em classes, de acordo com o tamanho de sua área nuclear, tendo por base o PI área nuclear dos fragmentos de floresta, e depois, estabelecidas as distâncias entre as classes de tamanho.

As áreas nucleares dos fragmentos foram divididas em classes, em cuja definição se consideraram apenas 
os fragmentos com área nuclear acima de 1 ha. A primeira classe estabelecida foi entre 1 e 9 ha (69 fragmentos), com maior frequência na bacia e mais distribuídos por sua paisagem, mas que são os de menor interesse e foram estabelecidas mais três classes com frequência de área nuclear menor, 10 - 49 ha (18 fragmentos), 50 - 75 ha (2 fragmentos) e 76 - 96 ha (1 fragmento). Logo em seguida, foi estabelecido que cada classe de área nuclear passou a constituir um novo mapa, a partir dos quais foram gerados mapas com distâncias entre as áreas nucleares dos fragmentos de floresta.

O próximo passo foi a normalização (padronização para uma única escala, nesse caso, variando de 0 a 255 bytes) desses mapas de distância, para posterior combinação de todos os mapas de fatores. Para normalização dos valores de distâncias, foi empregada uma função linear decrescente. Dessa forma, foram

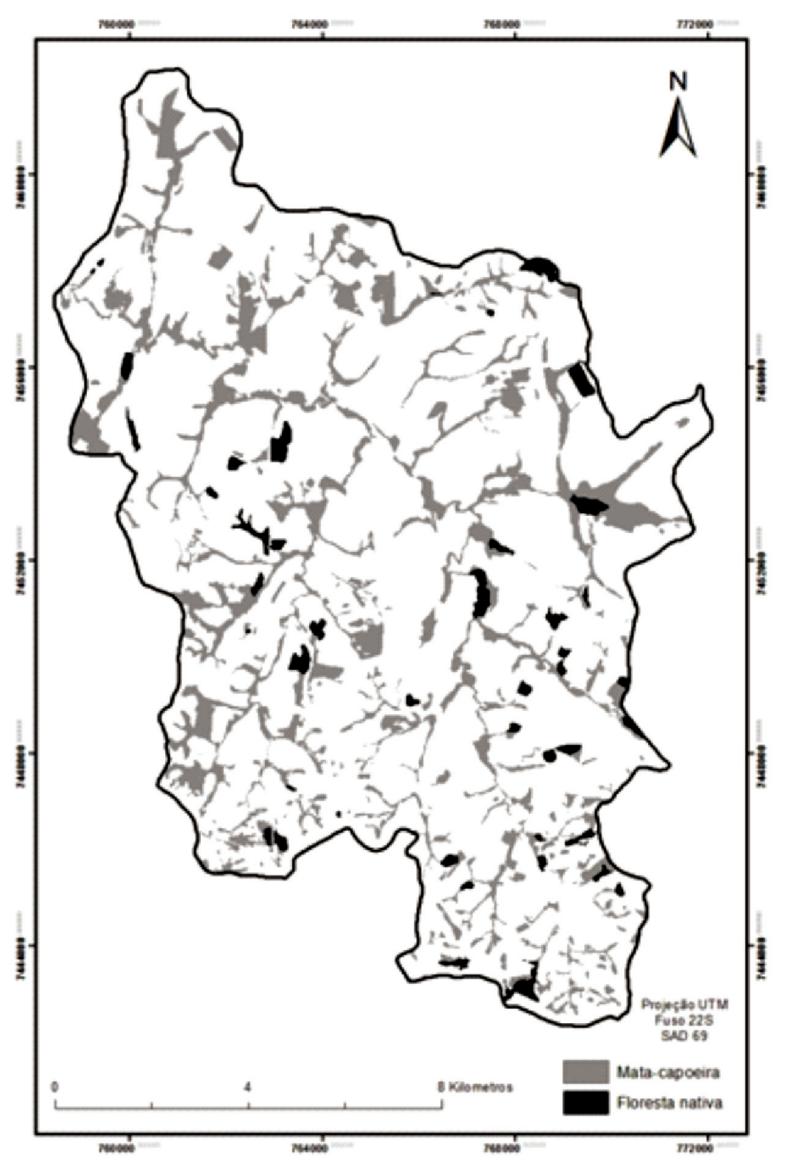

Figura 2 - Uso e cobertura do solo da Bacia do Rio Pardo. Figure 2 - Use and land cover in Pardo River Basin. obtidos os maiores valores (ex. 255), ou seja, maior prioridade, para as menores distâncias entre as áreas nucleares dos fragmentos.

Para compor o mapa de fator, todos esses mapas foram novamente unidos. Com essa união foram obtidos valores acima de 255 bytes, tornando necessária uma nova normalização de valor para gerar o mapa final. Dessa vez foi utilizada uma função linear crescente, que tem a propriedade de manter a escala de importância do mapa original.

(2) Proximidade da cobertura florestal: com este mapa de fator foram priorizadas as uniões entre os fragmentos de floresta nativa e mata capoeira, independentemente de seus tamanhos. A partir do PI, gerou-se um mapa com distâncias entre os fragmentos, sendo em seguida normalizado (escala 0 até 255 bytes) com função linear decrescente. Assim, ficou garantido que, quanto mais próximo da cobertura florestal, maior a importância (prioridade) da distância, estando os maiores valores (ex.: 255) associados aos fragmentos de floresta nativa e mata capoeira.

A distância entre os fragmentos de uma paisagem contribui para caracterização de sua configuração espacial e fornece indicativo sobre seu nível de fragmentação florestal (TUNER e GARDNER, 1990; YONG e MERRIAN, 1994; VALENTE, 2005).

(3) Proximidade da rede hidrográfica: para elaboração deste mapa de fator, produziu-se o mapa de distância dos corpos d'água, a partir do PI rede hidrográfica. O mapa final foi normalizado (escala 0 - 255 bytes) com função linear decrescente. Desse modo, obtiveram-se valores iguais e, ou, próximos a 255 na rede hidrográfica.

Foram considerados, na geração de áreas prioritárias para restauração florestal, apenas aspectos da paisagem, naturais ou antrópicos, desconsiderando-se a recomendação quanto à largura de faixas de Áreas de Preservação Permanente (APP), como consta do Código Florestal.

A proximidade da rede hidrográfica, pela importância da mata ciliar, foi um dos fatores adotados em avaliações multicritérios conduzidas por Dragan et al. (2003) e Ferraz e Vettorazzi (2003).

(4) Distância aos centros urbanos: a interferência humana, promovida pela relação de proximidade entre áreas urbanizadas e fragmentos

Revista Árvore, Viçosa-MG, v.36, n.6, p.1079-1090, 2012 
florestais, pode desfavorecer a conservação desses remanescentes, expondo-os a perturbações como incêndios, animais domésticos, caça, desmatamento e novos assentamentos urbanos e industriais.

As distâncias aos centros urbanos foram determinadas tendo por referência o PI centros urbanos. Como para esse fator quanto mais próximo a um centro urbano, menor a importância (prioridade) da distância, para sua normalização empregou-se uma função linear crescente.

Segundo Valente (2005), para a manutenção da biodiversidade e sucesso das ações de conservação e preservação florestal é interessante que sejam priorizadas as áreas mais distantes dos centros urbanos, por estarem mais preservados das ações antrópicas.

(5) Declividade: para elaboração desse mapa de fator, utilizou-se o PI com classes de declividade, em porcentagem, tendo sido obtido a partir da Modelagem Digital do Terreno. Para o objetivo deste trabalho, as áreas de maior declividade receberam níveis de importância (prioridade) mais elevados, pois são mais suscetíveis a processos erosivos. De acordo com Rosa et al. (2000), os processos erosivos em uma bacia hidrográfica podem impactar sensivelmente a conservação de fragmentos florestais.

A produção desse fator foi obtida com uma função linear crescente, sendo, assim, a imagem desse fator normalizada $(0-255$ bytes $)$ de maneira a ter maior prioridade para os maiores valores de declividade.

(6) Erodibilidade do solo: oo mapa do fator foi obtido a partir da reclassificação do mapa de solos, sendo cada classe de solo associada ao valor correspondente de erodibilidade. A erodibilidade do solo tem seu valor quantitativo determinado experimentalmente em parcelas unitárias, sendo expresso como a perda de solo por unidade de índice de erosão da chuva, tendo por unidade $\mathrm{Mg}$ ha $\mathrm{h} \mathrm{ha}^{-1} \mathrm{MJ}^{-1} \mathrm{~mm}^{-1}$ (BERTONI e LOMBARDI NETO, 1985).

A imagem de fator erodibilidade do solo foi submetida a uma função linear crescente, padronizada para 0 a 255 bytes. Desse modo, obtiveram-se os valores iguais e, ou, próximos a 255 para a classe de solo com maior índice de erodibilidade.

A erodibilidade foi utilizada diretamente como um fator, em análises espaciais, envolvendo vários critérios, como em Adinarayana et al. (1999) e Richardson e Gatti (1999).

Revista Árvore, Viçosa-MG, v.36, n.6, p.1079-1090, 2012
(7) Restrições:Foram foram consideradas como restrições para o propósito deste trabalho as áreas de floresta nativa, os corpos d'água e as áreas urbanas, ou seja, todas as áreas onde não foi considerada a possibilidade de restauração da cobertura florestal.

\subsection{Pesos dos mapas de fatores}

Para calcular o peso de cada fator foi utilizado o processo de tomada de decisão conhecido como Análise HierárquicaAnalítica (SAATY, 1977). Esse método emprega uma comparação pareada entre fatores para determinar a importância relativa de cada um deles (CONINE et al., 2004; SILVEIRA et al., 2008).

A atribuição de pesos aos critérios consiste em definir a quantificação da importância de cada um deles no processo de decisão. Vários métodos foram desenvolvidos para auxiliar a definição dos pesos de compensação dos fatores, entre eles o da ordenação, da escala de pontos, da distribuição de pontos e do método baseado na comparação de critérios dois a dois (RAMOS e MENDES, 2001).

Para elaboração da matriz de comparação, os fatores foram comparados, dois a dois, utilizando como referência Eastman (2001), a escala contínua de nove pontos (Tabela1). Os pesos de compensação, que expressam a importância, ou ordem de importância dos fatores no processo de tomada de decisão, foram determinados com base na revisão de literatura, em projetos desenvolvidos na Técnica Participatória.

Devido ao fato de a matriz de comparação pareada apresentar múltiplos caminhos (ou maneiras), pelos quais a importância relativa dos critérios pode ser avaliada, é possível também determinar o grau de consistência, atingido no desenvolvimento dos pesos. Saaty (1980) indicou o procedimento pelo qual uma Taxa de Consistência (TC) pode ser obtida. Ela mede a intensidade ou grau da inconsistência em uma matriz de julgamentos paritários e avalia quanto o maior autovalor dessa matriz se afasta da ordem da matriz. O cálculo do Índice de Consistência (IC) é:

$$
\mathrm{IC}=|(\lambda \max -\mathrm{N})| /(\mathrm{N}-1)
$$

onde: $\mathrm{N}$ e $\lambda$ max representam, respectivamente, a ordem e o estimador de autovalor máximo da matriz de julgamentos paritários.

Revista Árvore, Viçosa-MG, v.36, n.6, p.1079-1090, 2012 
Segundo Saaty (1980), as matrizes com TC maiores que 0,10 devem ser reavaliadas, pois a inconsistência é um fato inerente ao ser humano, portanto deve existir tolerância para sua aceitação. Vargas (1982) propôs a aceitação de julgamentos que gerem inconsistência com TC inferior a 0,1 .

\section{6.Áreas prioritárias para restauração florestal geradas pelo método da Combinação Linear Ponderada}

O mapa de áreas prioritárias para restauração florestal foi gerado pelo método da Combinação Linear Ponderada, através da análise integrada dos dados disponíveis sobre a área em estudo, segundo os critérios e pesos estabelecidos pela Técnica Participatória - Análise Hierárquica Analítica.

O mapa final de áreas prioritárias foi reclassificado para melhor avaliar a relação das áreas prioritárias à restauração florestal, além de tornar mais fácil a interpretação do mapa. Foram definidas para o mapa de áreas prioritárias cinco classes de prioridades (classes de mesma amplitude): muito baixa, baixa, média, alta e muito alta. $\mathrm{O}$ intervalo de classe foi determinado a partir da avaliação do histograma dos mapas (0-255 bytes) e definição dos limites inferiores e superiores de variação por divisão linear simples dos 255 níveis do histograma em cinco classes. Essas classes significam o nível de importância e prioridade, em que as classes muito alta e alta possam ser contempladas por ações que visam favorecer a conectividade entre os fragmentos florestais.

\section{RESULTADOS}

Considerando que as variáveis que interferem na escolha de áreas prioritárias à restauração florestal na Bacia do Rio Pardo-SP contribuem com pesos diferenciados no processo final de decisão, estabeleceuse uma hierarquia, de acordo com a importância de cada fator para a aptidão da área. Os pesos e a prioridade dos respectivos fatores estão representados na matriz de comparação pareada entre os fatores (Tabela 2).

O fator de maior peso foi a proximidade entre fragmentos de maior área nuclear $(0,3713)$, seguido de proximidade da cobertura florestal $(0,1911)$, proximidade da rede hidrográfica $(0,1516)$, distância aos centros urbanos $(0,1168)$, declividade $(0,0840)$ e erodibilidade $(0,0854)$. Deve-se ressaltar que o ranqueamento e os pesos dos fatores foram definidos com aplicação da Técnica Participatória - Análise Hierárquica Analítica, que consiste na reunião entre especialistas nas diferentes áreas de interesse para o projeto. Essa ponderação teve influência direta sobre a espacialização das áreas prioritárias à restauração florestal a serem geradas pela análise.

A taxa de consistência (TC) obtida para a matriz foi de 0,04 (menor que 0,10 ), que indica que os valores de comparação entre os fatores foram gerados aleatoriamente, portanto não houve a necessidade de reorganização da matriz.

A Figura 3 mostra o mapa de áreas prioritárias à restauração florestal na Bacia do Rio Pardo-SP, gerado através da análise integrada dos dados disponíveis sobre a área em estudo, segundo os critérios e pesos estabelecidos. Pode-se observar que o mapa não identificou as áreas aptas ou inaptas, mas representou superfície de aptidão.

A partir da superfície de aptidão, foi possível efetuar uma hierarquização das células de forma a selecionar a melhor área (classe muito alta), com 1.502,39 ha (10\%), conforme quantificado na Tabela 3 . A seleção de áreas de prioridade muito alta permitiu a conexão de uma ou mais áreas contíguas, favorecendo a estabilidade dos fragmentos próximos uns dos outros unidos. A Figura 3 detalha a disposição dos fragmentos na classe muito alta. Dessa forma, o mapa permitiu uma visão geral de como a paisagem da bacia se comporta em termos de aptidão à implantação de ações de restauração florestal.

Tabela 1 - Escala contínua para elaboração da matriz de comparação pareada.

Table 1 - The continuous rating scale used for preparing the pair-wise comparison matrix.

\begin{tabular}{cccccccc}
\hline $1 / 9$ & $1 / 7$ & $1 / 5$ & $1 / 3$ & 1 & 3 & 7 & 9 \\
\hline $\begin{array}{c}\text { Extremamente } \\
\text { fortemente }\end{array}$ & $\begin{array}{c}\text { Muito } \\
\text { fortemente }\end{array}$ & Moderadamente & Igualmente & Moderadamente & Fortemente & Muito Extremamente \\
fortemente & & & & &
\end{tabular}

Fonte: Eastman, 2001. 
Tabela 2 - Pesos de compensação obtidos através da matriz de comparação pareada.

Table 2 - Weights compensation obtained by comparison pair wise matrix.

\begin{tabular}{lccccccc}
\hline & Fator 1 & Fator 2 & Fator 3 & Fator 4 & Fator 5 & Fator 6 & Pesos \\
\hline Fator 1 & 1 & - & - & - & - & - & 0,0854 \\
Fator 2 & 1 & 1 & - & - & - & - & 0,0840 \\
Fator 3 & 1 & 2 & 1 & - & - & - & 0,1168 \\
Fator 4 & 2 & 2 & 2 & 1 & - & - & 0,1516 \\
Fator 5 & 2 & 2 & 2 & 2 & 1 & - & 0,1911 \\
Fator 6 & 5 & 3 & 2 & 3 & 3 & 1 & 0,3713 \\
\hline
\end{tabular}

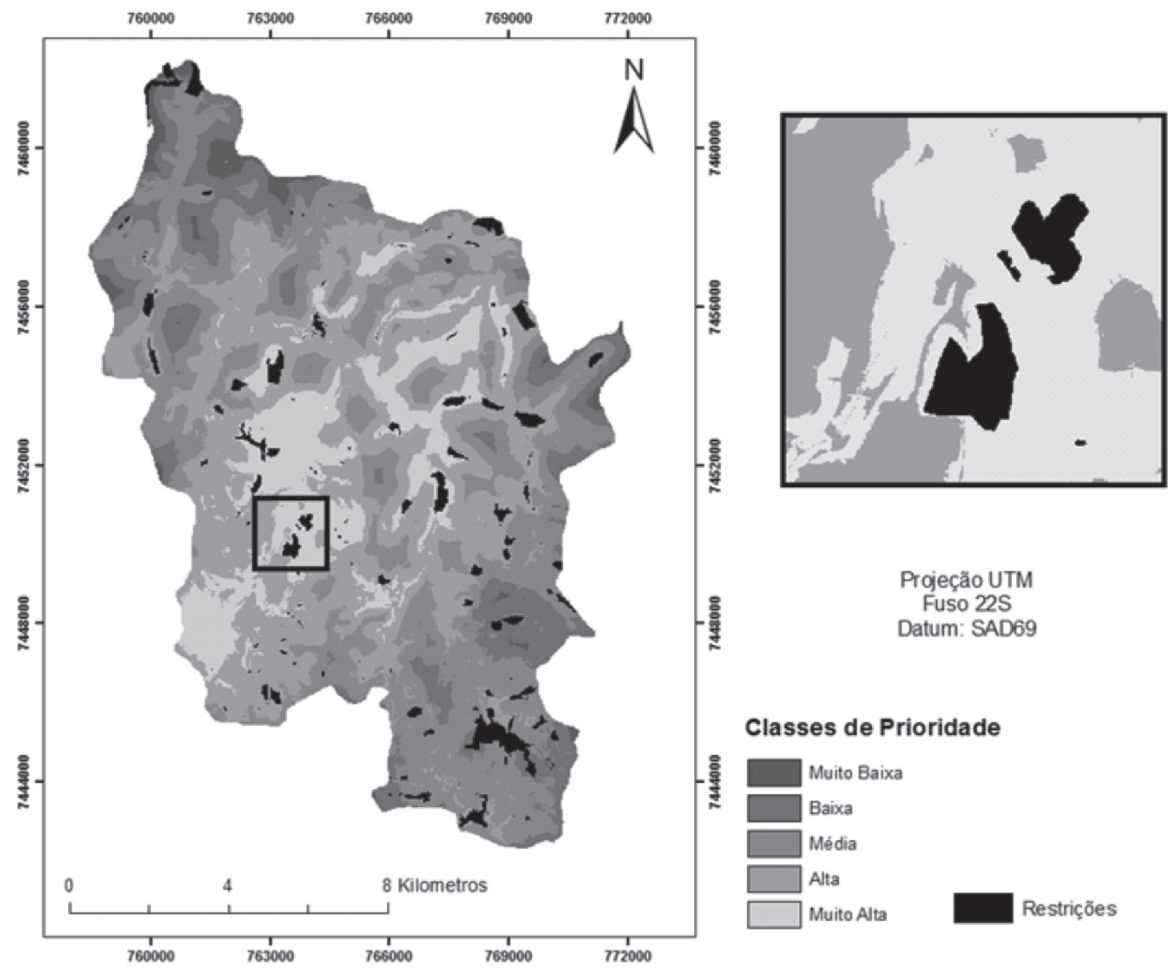

Figura 3 - Áreas prioritárias à restauração florestal geradas pelo método da Combinação Linear Ponderada (cinco níveis de prioridade).

Figure 3 - Priority areas for forest restoration generated by the method Weighted Linear Combination (five levels of priority).

\section{DISCUSSÃO}

Foi verificado em campo que grande parte da vegetação nativa foi suprimida para dar espaço às atividades agropecuárias e, em alguns casos, para o reflorestamento com espécies exóticas e, também, devido à expansão da cana-de-açúcar. Existem muitas áreas de mata em regeneração, principalmente em áreas de borda dos fragmentos de floresta, com muitas espécies pioneiras, típicas de capoeira, dada a proximidade das áreas urbanas e utilizadas com agropecuária durante muitos anos. Poucas áreas vegetadas na Bacia do Rio Pardo são matas "primárias" e, dada a dificuldade de diferenciação nas imagens, optou-se pela inclusão de capoeiras à classe mata capoeira. O mapa de uso do solo da Bacia do Rio Pardo em classes de mata capoeira e floresta nativa (Figura 1) tornou-se mais fácil compreender as ações de recuperação florestal na Bacia do Rio Pardo-SP, utilizando a abordagem multicriterial.

Revista Árvore, Viçosa-MG, v.36, n.6, p.1079-1090, 2012 
Tabela 3 - Valores de área para os cinco níveis de prioridade para restauração florestal.

Table 3 - Area values for the five priority levels for forest restoration.

\begin{tabular}{ccc}
\hline Nível de prioridade & \multicolumn{3}{c}{ Área } \\
\hline & $($ ha) & $(\%)$ \\
Muito baixo & 19,69 & 0,13 \\
Baixo & 842,09 & 5,68 \\
Médio & $5.227,62$ & 35,27 \\
Alto & $7.229,83$ & 48,78 \\
Muito alto & $1.502,39$ & 10,14 \\
\hline Total & $14.821,62$ & 100,00 \\
\hline
\end{tabular}

O mapa de áreas prioritárias com cinco classes de prioridade representou a melhor caracterização da paisagem no processo de tomada de decisão e possibilitou o melhor entendimento entre as alterações necessárias na estrutura da paisagem e as classes de prioridade ("ordem de prioridades").

Foi possível esse detalhamento, em especial da classe de maior prioridade, em função dos pesos de ordenação atribuídos aos fatores. Jiang e Eastman (2000) também comprovaram a possibilidade de aumento no número de classes à medida que se obtêm soluções mais arriscadas.

A priorização de áreas na bacia, de acordo com esse mapa, favoreceu a conexão e a melhoria na forma dos maiores fragmentos com área nuclear. Observouse que existe correspondência entre a concentração de fragmentos de floresta com área nuclear e as classes de valores da escala de prioridades $(0-255$ bytes $)$. As regiões da bacia onde esses fragmentos são praticamente inexistentes foram associadas aos menores valores de prioridade ( 0 byte), e, em contrapartida, as marcadas pela maior concentração desses fragmentos foram associadas aos maiores valores de prioridade (255 bytes).

Para fragmentos de floresta com área nuclear próximos uns dos outros, como ocorre com aqueles localizados na classe de maior prioridade (Figura 3), haverá melhorias nos fatores que afetam a conservação da biodiversidade em fragmentos florestais, como: tamanho, forma, grau de isolamento, tipo de vizinhança e histórico de perturbações, desde que haja a conexão entre eles.

Com a conexão entre os fragmentos com maior área nuclear, segundo Lathrop et al. (1998) e Geneletti
(2004), favorecendo ações de conservação e preservação florestal, essa conexão contribui para a reestruturação dos componentes básicos da estrutura de uma paisagem e visa manter a integridade de sua cobertura florestal natural.

Ressalta-se a importância do fator proximidade entre fragmentos para o mapa de prioridades. Metzger (2003) enfatizou a importância da proximidade entre fragmentos na conservação biológica, além de que os grandes fragmentos e os fragmentos menores promovem a conectividade funcional da paisagem. Esse fator, juntamente com o fator proximidade entre fragmentos de maior área nuclear, possibilitou a definição de grande parte das áreas aptas à revegetação com floresta nativa.

Outra característica a ser ressaltada, ainda, no mapa de áreas prioritárias é a continuidade de suas classes de prioridade. A categoria de maior prioridade acaba sendo conectada pela categoria seguinte (ordem de menor prioridade), e assim sucessivamente.

Para o planejamento de recomposição florestal de toda a Bacia do Rio Pardo seria conveniente a união de outras áreas regionais prioritárias no seu entorno.

\section{CONCLUSÕES}

AAvaliação Multicritérios em ambiente SIG mostrouse flexível, fácil de ser implementada, e permitiu a interação entre as dimensões humanas (conhecimento científico) e características biofísicas da paisagem, em um processo de tomada de decisão. A metodologia mostrou-se adequada ao mapeamento de áreas prioritárias à restauração florestal em bacias hidrográficas, visando favorecer a conexão entre os fragmentos florestais. Para utilização desse método, deve-se ter bom conhecimento da paisagem a ser estudada para que se definam com coerência os pesos e ordenação atribuídos aos fatores.

As informações geoespaciais sobre o meio biofísico, geradas a partir dos procedimentos metodológicos descritos neste artigo, têm alto potencial para favorecer a conexão entre os fragmentos florestais, visando a um planejamento racional do uso dos recursos naturais e a ocupação territorial, além de servir como forte instrumento de orientação às políticas públicas e aos processos coletivos de decisão.

Salienta-se que a proposta desta metodologia, onde a seleção e a definição dos critérios e seus respectivos

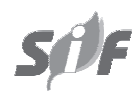

Revista Árvore, Viçosa-MG, v.36, n.6, p.1079-1090, 2012 
pesos referentes a cada um podem variar de acordo com as características da área, com o interesse e objetivo do estudo, possibilitou a integração dos aspectos da paisagem na determinação das áreas prioritárias à restauração florestal na Bacia do Rio Pardo-SP.

\section{AGRADECIMENTOS}

Ao Conselho Nacional de Desenvolvimento Científico e Tecnológico (CNPq), pelo apoio financeiro concedido ao primeiro autor; e ao Grupo de Estudos e Pesquisas Agrárias Georreferenciadas (GEPAG) - FCA/ UNESP, pelos ensinamentos e pela saudável amizade.

\section{REFERÊNCIAS}

ADINARAYANA, J.et al.A rule-based soil erosion model for a hilly catchment. Catena, v.37, n.3/4, p.309-318, 1999.

\section{ALMEIDA, F. F. M.Fundamentos} geológicos do relevo paulista. São Paulo: Instituto de Geografia, Universidade de São Paulo, 1964. 99p.

BERTONI, J.; LOMBARDI NETO, F. Conservação do solo. Piracicaba: Livroceres, 1985. 368p.

CARVALHO, W.A.; JIM, J. Áreas de proteção ambiental: Região da "Serra de Botucatu" e Região da "Serra de Fartura". Botucatu: Instituto Básico de Biologia, Faculdade de Ciências Agronômicas, Universidade Estadual Paulista, 1983. 47p.

CHEN, K. P.; BLONG, R.; JACOBSON, C. MCERISK: integrating multicriteria evaluation and GIS for risk decision-making in natural hazards. Environmental Modeling e Software, v.16, n.4, p.387-397, 2001.

CONINE, A. et al. Planning for multipurpose greenways in Concord, North Carolina.

LandscapeUrban Planning,n. 68, p.271287, 2004.

CONTE, M. L. Aspectos quantitativos e qualitativos das águas da Bacia Experimental do Rio Pardo - Região de Botucatu, SP. 1999.157f.Tese (Doutorado em Agronomia) Faculdade de Ciências Agronômicas, Universidade Estadual Paulista,Botucatu, 1999..
DRAGAN, M. et al. Application of a spatial decision support system (SDSS) to reduce soil erosion in northern Ethiopia.

EnvironmentalModelling \& Software, v.18, n.10, p.861-868, 2003.

EASTMAN, J. R. Decision support: decision strategy analysis. In: IDRIS 32 release 2: guide to GIS and image processing. Worcester: Clark University/Clark Labs, 2001. v.2.p.1-22.

FERRAZ, S. F. B.; VETTORAZZI, C. A. Identificação de áreas para recomposição florestal com base em princípios de Ecologia da Paisagem. Revista Árvore, v.27, n.4, p.575-583, 2003.

GENELETTI, D. Using spatial indicators and value functions to asses ecosystem fragmentation caused by linear infrastructures. International Journal of Applied Earth Observation and Geoinformation, v.5, p.1-15, 2004.

GROSSI, C. H. Sistema e informação geográfica - Basins 3.0 na modelagem hidrológica da Bacia Experimental do Rio Pardo, SP. 2003. 101f. Dissertação (Mestrado em Agronomia) - Faculdade de Ciências Agronômicas, Universidade Estadual Paulista, Rio Pardo, 2003.

JIANG, H.; EASTMAN, J. R. Application of fuzzy measures in multi-criteria evaluation in GIS.

International Journal of Geografhical Information Science, v.14, n.2, p.173-184, 2000.

LATHROP, R. G.; BOGNAR, J. A. Applying GIS and landscape ecological principles to evaluate land conservation alternatives. Landscape and Urban Planning,v.41, p.27-41, 1998.

LORD, J. M.; NORTON, D. A. Scale and the spatial concept of fragmentation. Conservation Biology, v.2, n.4, p.197-262, 1990.

METZGER, J. P. Como restaurar a conectividade de paisagens fragmentadas? In: KAGEYAMA, P. Y.et al.Restauração ecológica de ecossistemas naturais. Botucatu: FEPAF, 2003. p.51-76.

MALCZEWSKI, J. GIS and multicriteria decision analysis. New York: John Wiley, 1999. 362p. 
MALCZEWSKI, J. GIS-based multicriteria decision analysis: a survey of the literature.

International Journal of Geographical Information Science, v.20, n.7, p.703-726, 2006,

MURCIA, C. Edge effects in fragmented forests: implications for conservation. Trends in Ecology and Evolution, v.10, n.1, p.58-62, 1995.

NOSS, R. F.; O'CONNEL, M. A.; MURPHY, D. D. The science of conservation of planning: habit-based conservation under the endangered species. Washington DC: Act. Island Press, 1997. 154p.

PUTZ, F. E. et al. Tropical forest management and conservation of biodiversity: an overview. Conservation Biology, v.1, n.15, p.7-20, 2001 .

PUTZ, S.et al.Fragmentation drives tropical forest fragments to early successional states: A modelling study for Brazilian Atlantic forests.EcologicalModelling, v.222, n.12, p.1986-1997, 2011.

RAMOS, R. A. R.; MENDES, J. F. G. Avaliação de aptidão do solo para localização industrial: ocaso de Valença. RevistaEngenharia Civil, v.10, n.1, p.7-29, 2001 .

RICHARDSON, M.S.; GATTI, R.C. Prioritizing wetland restoration activity within a Wisconsin watershed using GIS modeling. Journal of Soil and Water Conservation, v.54, n.3, p.537-542, 1999.

ROSA, D. et al. Assessment of soil erosion vulnerability in western Europe and potential impact on crop productivity due to loss of soil depth using the Impelero model. Agriculture, Ecosystems and Environment, v.81, n.2, p.179-190, 2000.

SAATY, L. L. A scaling method for priorities in hierarchical structures.Journal ofMathematical Psychology, v.15, n.3, p.234-281, 1977.

SAATY, T. The analytic hierarchy process. New York: McGraw-Hill, 1980. 287p.
SARTORI, A. A. C.Análise multicritérios na definição de áreas prioritárias à conectividade entre fragmentos florestais.2010. 98f.Dissertação (Mestrado em Agronomia) - Faculdade de Ciências Agronômicas, Universidade Estadual Paulista, Botucatu,2010.

SARTORI, A. A. C.; ZIMBACK, C. R.

L.Recomposição florestal visando à conservação de recursos hídricos na bacia dorio Pardo, SP.

Revistade Energia na Agricultura, v.26, n.4, p.43-53, 2011.

SILVEIRA, H. L. F.; VETTORAZZI, C. A.; VALENTE, R. O. A.Avaliação multicriterial no mapeamento de risco de incêndios florestais, em ambiente SIG, na bacia do Rio Corumbataí, SP. Revista Árvore, v.32, n.2, p.259-268, 2008.

SOUZA, A. J.et al. Aspectos físicos do município de Botucatu. Botucatu: Pólo de Desenvolvimento Regional, 1985.34p. (Não Publicado)

TORNERO, M. T. Fotointerpretação da cobertura vegetal e da rede de drenagem da bacia experimental do Rio Pardo, no período de 15 anos. 1996. 129f.Dissertação (Mestrado em Agronomia - Energia Agricultura) UNESP,Faculdade de Ciências Agronômicas, 1996.

TURNER, M. G.; GARDNER, R. H. Quantitativemethods in landscapeecology: theanalysisandinterpretationoflandscapeheterogeneity. New York: Springer Verlag, 1990. 536p.

VALENTE, R. O. A. Definição de áreas prioritárias para conservação e preservação florestal por meio da abordagem multicriterial em ambiente SIG. 2005. 121f. Tese (Doutorado - Recursos Florestais) - Escola Superior de Agricultura "Luiz de Queiroz" USP, Piracicaba,2005. 121 p.

VARGAS, G. L. Reciprocal matrices with random coefficients. Mathematical Modelling, v.3, n.1, p.69-81, 1982.

VETTORAZZI, C. A. Avaliação multicritérios, em ambiente SIG, na definição de áreas prioritárias à restauração florestal visando à conserva de recursos hídricos. 2006. 151f. Tese (Livre Docência na Especialidade/Disciplina Topografia) - Escola Superior de Agricultura "Luiz de Queiroz" USP, Piracicaba,2006.

Revista Árvore, Viçosa-MG, v.36, n.6, p.1079-1090, 2012 
VIANA, V.M. Conservação da biodiversidade de fragmentos de florestas tropicais em paisagens intensivamente cultivadas. In: ABORDAGENS interdisciplinares para a conservação da biodiversidade e dinâmica do uso da terra no novo mundo. Belo Horizonte/Gainesville: ConservationInternational do Brasil/Universidade Federal de Minas Gerais/ Universityof Florida, 1995. p.135-154.

VIANA, V. M.; PINHEIRO, L. A. F. V. Conservação da biodiversidade em fragmentos florestais.Série Técnica IPEF, v.12, n.32, p.25-42, 1998.

VOOGD, H. Multicriteria evaluation for urban and regional planning. London: Pion, 1983. 370p.
WANG, D. H.; MEDLEY, K. E. Land use model for carbon conservation across a Midwestem USA landscape. Landscape and Urban Planning, v.69, n.4, p.451-465, 2004.

YONG, A. G.; MERRIANM, H. G. Effects of forest fragmentation on the spatial genetic structure of Acer sacvharum Marsh. (sugar maple) population. Heredity, v.1, NUMERO,p.277-289, 1994.

ZIMBACK, C. R. L. Levantamento semidetalhado dos solos da bacia do Rio Pardo no Municípios de Pardinho e Botucatu. Botucatu: Faculdade de Ciências Agronômicas, Universidade Estadual Paulista, 1997. 55p. 